\title{
Effect Of Artesunate, Esomeprazole And Coffee Intake On Prodromal Parkinson's Disease Features In Adult Men
}

\author{
Stephen E Oriaifo ${ }^{1}$, Isa M Abdul-Aguye ${ }^{2}$, Hubert O Omeife ${ }^{3}$, Ray I Ozolua ${ }^{4}$ \\ ${ }^{I}$ Dept of Pharmacology, Ambrose Alli University, Ekpoma, Edo State, Nigeria, ${ }^{2}$ Dept of Pharmacology, Ambrose Alli \\ University, ABU, Zaria, Nigeria, ${ }^{3}$ Dpet of Medicine, ISTH, Irrua, Nigeria, ${ }^{4}$ Dept of Pharmacology, Ambrose Alli \\ University, UNIBEN, Benin-City, Nigeria
}

\section{Background}

The convergent molecular mechanisms of aging, type 2 diabetes mellitus, Alzheimer's disease, Parkinson's disease and Huntington's disease offer druggable targets. Present drugs for treatment of Parkinson's disease (PD) often fail to maintain steady efficacy and are often associated with undesirable side- effects There is now a paradigm shift in this agerelated neurodegenerative disorder to agents that co-operatively target immune activation, the noxious agents-induced ATP-P2X7 cell death receptor-NLRP3inflammasome-adenosine A2A receptor-HMGBI signalling, upregulate the antioxidant Nrf-2/ARE axis, insulin signalling, and enhance mitochondrial function to attenuate the hallmarks of this intriguing disease.

Methods

Animal experimentations were done using male adult mice administered artesunate $(30 \mathrm{mg} / \mathrm{kg})$ and caffeine $(6 \mathrm{mg} / \mathrm{kg}) \mathrm{i}$. p. on alternate days, after ethical approval in the University of Benin, Benin-City. Effect of this treatment in lowering glucose and uric acid levels in ad libitum high-lipid + low-dose streptozotocin-induced type 2 diabetic male mice compared to controls was investigated. In the clinical work, the effect of low-dose artesunate (12.5 mg daily) and low-dose esomeprazole (10 mg daily) use in conjunction with coffee intake was studied in 5 elderly patients with early-onset (prodromal) Parkinson's disease who had non-motor symptoms such as REM sleep disturbance, constipation; and the motor symptoms of bradykinesia, tremors, rigidity and postural imbalance.

Results

Experiments demonstrated that artesunate has a significant additive effect $(\mathrm{P}<0.05)$ with caffeine in lowering glucose and uric acid levels in streptozotocin-induced type 2 diabetic male mice compared to caffeine alone. 3 weeks institution of the combined therapy significantly $(\mathrm{P}<0.05)$ abrogated the prodromal non-motor and motor features of Parkinson's disease.

Conclusion

The implication of the present study is that the combined remedies deserve more attention in the prevention of the progression and treatment not only of Parkinson's disease but also of other age-related neurodegenerative disorders.

KEYWORDS: Parkinson's disease; Prodromal Stage; Coffee; Esomeprazole; Artesunate 